\title{
The Effect of Sample Size on Crown Size Asymmetry
}

\author{
S. M. GARN, P. E. COLE and B. H. SMITH \\ Center for Human Growth \& Development, The University of Michigan, Ann Arbor, Michigan 48109
}

J Dent Res 58(10): 2012, October 1979

There is now considerable interest in dental asymmetry, whether from an evolutionary standpoint or with respect to environmental stresses. The recent literature contains suggestions that dental asymmetry may change over evolutionary time (Suarez, Am J Phys Anthrop, 41:411-416, 1974; Wolpoff, Am $J$ Phys Anthrop, 43:307-308, 1975), or increase as a function of environmental stress (Perzigian, Am J Phys Anthrop, 47:81-88, 1977), or be affected by inbreeding (Niswander and Chung, Am J Hum Genet, 17:390-398, 1965).

However, it is also apparent that the magnitude of asymmetry, whether measured as RMS asymmetry (the standard deviation of the left-right difference) or as left-right correlations ( $r)$, is subject to the exigencies of sampling. Very high and very low values are more likely with small sample sizes, along the lines previously noted for intertooth correlations (Garn, Cole and Guire, $J$ Dent Res, 56:1474, 1977), bodily dimensions during growth (Garn et al., Ecol Food Nutr, 6:153-157, 1977), and parent-child stature correlations (Garn and Bailey, in Human Growth, ed. by Falkner and Tanner, 1978, p. 311). Since the effect of sample size on left-right dental asymmetry has not been studied, and since some reported differences in RMS asymmetry may be due to small sample size, we attempt to clarify the effects of sampling here.

Basic statistics on individual left-right crown size asymmetry were calculated on a combined sample from three different data bases previously described by us. These include the Fels Institute longitudinal series sample of 656 individuals (Garn, Lewis and Walenga, Archs Oral Biol, 13: 841-844, 1968), the University of Michigan longitudinal series of 208 (Moyers et al., Standards of Human Occlusal Development, 1976, pp. 7-10), and the NINCD\&S Collaborative Perinatal Project subseries of 2240 individuals (Garn, Osborne and McCabe, Am $J$ Phys Anthrop, 51: in press, 1979). From the total series, with sample sizes as great as 1322 observations for specific pairs of teeth, 11 independent random subsamples of $1000,900,750,625,400,225,100,75,50$, 25 , and 15 were selected by a computer subroutine of the MIDAS statistical analysis program (Fox and Guire, Documentation for MIDAS, The Statistical Research Laboratory, The University of Michigan, 1976 , p. 82). The random samples were succes-

Received for publication February 9, 1979. Accepted for publication April 11, 1979.

sively drawn (with replacement) from the total sample for each tooth. Mean left-right differences $\left(\overline{\mathrm{X}}_{\mathrm{d}}\right)$ and RMS asymmetry were calculated for each of the 11 random samples for each of the 14 permanent teeth $\left(I^{1}-M^{2}\right.$ and $\left.I_{1}-M_{2}\right)$.

The maximum and minimum values of RMS asymmetry among the 14 teeth for each of the 11 declining subsamples are shown in the figure. It is obvious that the maximum and minimum values of RMS asymmetry are associated with the smallest sample sizes $(\leq 50)$, and that the range of obtained asymmetry values decreases by more than half with increasing sample size. As can be seen from the figure, little reduction in the range of obtained RMS asymmetry is attained beyond the sample size of 625 . However, with independentlydrawn sample sizes below 400 RMS asymmetry increases with decreasing values of $\mathrm{N}$.

Since most published studies on left-right dental asymmetry are based on small samples (generally below 100 and in some cases as small as 15), it is increasingly likely that apparent intergroup differences in crown asymmetry may reflect sampling limitations.

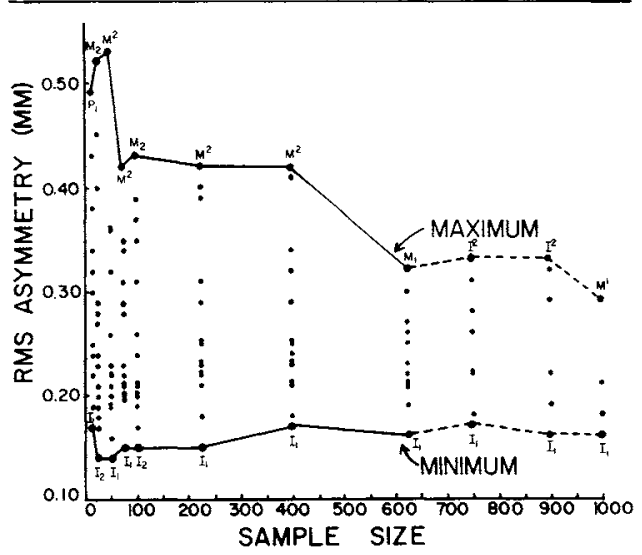

Effect of sample size (abscissa) on range of RMS (root mean square) asymmetry values (ordinate) for 11 successively-larger subsamples for 14 permanent teeth. As shown by the outer lines, the range of left-right crown-size asymmetry tends to become smaller as sample size increases, stabilizing after an $\mathrm{N}$ of 625 . 